\title{
Hallazgos ecocardiográficos en trabajadores de salud recuperados de infección leve por SARS- CoV-2 de un hospital IV COVID del Perú
}

\section{Echocardiographic findings in health workers recovered from mild infection by SARS-CoV-2 from a COVID IV hospital in Peru}

Correspondencia Roberto Baltodano-Arellano rbaltodanoa@unmsm.edu.pe

Recibido: 25/06/2021 Aprobado: 27/12/2021

Citar como: Baltodano-Arellano $R$, Cupe-Chacalcaje K, Rojas $P$, Meneses $G$, UrdaniviaRuiz D, Rafael-Horna E, Falcón-Quispe L, CachicatariBeltrán A, Hurtado-Belizario K, Levano-Pachas $G$. Hallazgos ecocardiográficos en trabajadores de salud recuperados de infección leve por SARS-CoV 2 de un hospital IV COVID del Perú. Acta Med Peru. 2021; 38(4): 337-40. doi: https://doi.org/10.35663/ amp.2021.384.2066
Roberto Baltodano-Arellano 1,2,a,b , Kelly Cupe-Chacalcaje 1,a , Paol Rojas ${ }^{1, a}$, Giovanni Meneses 2,b , Dante Urdanivia-Ruiz 1,a , Eliana Rafael-Horna 1,a , Luis Falcón-Quispe 1,a , Angela Cachicatari-Beltrán 1,a , Karla Sue América Hurtado-Belizario ${ }^{1, a}$, Gerald LevanoPachas ${ }^{1, a}$

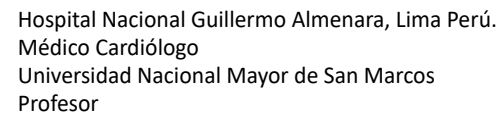

\section{Sr. Editor:}

La pandemia originada por el SARS-CoV-2 (Severe Acute Respiratory Syndrome-Coronavirus-2) representa un gran desafío para los sistemas de salud de todo el mundo. Su altísima contagiosidad ha generado

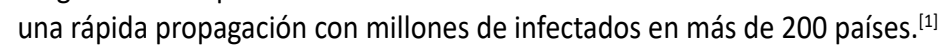

Aunque la infección por el SARS-CoV-2 produce primordialmente un compromiso pulmonar, otros órganos como el corazón, el cerebro y riñones pueden comprometerse de forma importante. Se ha reportado hasta un $27 \%$ de compromiso miocárdico en pacientes hospitalizados, lo que confiere peor pronóstico. ${ }^{[2]}$ Una cohorte de recuperados estudiados por resonancia cardiaca evidenció afectación miocárdica hasta en el $78 \%$ de los casos, independiente de las condiciones preexistentes y gravedad de la enfermedad. ${ }^{[3]}$

La exploración ecocardiográfica ha sido de utilidad para evaluar el compromiso cardiaco en la infección por SARS-CoV-2, tanto en la fase activa como en recuperación ${ }^{[4]}$, sin embargo, existe información limitada sobre el efecto cardiaco a corto y largo plazo. Planteamos un estudio transversal en un grupo de trabajadores de salud del Hospital Nacional Guillermo Almenara Irigoyen (Lima, Perú) recuperados de infección por SARS-CoV-2 con el objetivo de determinar hallazgos ecocardiográficos indicativos de secuelas cardíacas estructurales y/o funcionales.

Se incluyeron trabajadores de salud (personal asistencial y administrativo) voluntarios recuperados de la infección por SARS-CoV-2. Los criterios de inclusión fueron la infección confirmada mediante prueba molecular (PCR), prueba rápida (detección de anticuerpos $\operatorname{lgM} / \mathrm{lgG}$ ) o patrón tomográfico típico (infiltración en vidrio esmerilado). Fueron excluídos sujetos con mala ventana ecocardiográfica. Para determinación de severidad clínica se utilizó la clasificación de Wuhan. Los estudios de ecocardiografía convencional fueron realizados por dos operados certificados en imagen cardíaca, utilizando un ecógrafo comercial (Vivid E95 GE) y siguiendo las medidas de seguridad según el contexto de pandemia y recomendaciones internacionales. Para el análisis de las variables cuantitativas se utilizaron la media y desviación estándar, o la mediana y rango intercuartil. Las variables cualitativas se mostraron como frecuencias y porcentajes. 
Tabla 1. Características clínicas y ecocardiográficas de voluntarios recuperados de SARS-CoV-2 leve. Hospital IV COVID, Perú

\section{Característica}

\section{Características Clínicas}

Edad (años)

64

37,7

Peso (Kg)

64

66,8

13,5

Talla $(\mathrm{cm})$

158,8

IMC $\left(\mathrm{Kg} / \mathrm{m}^{2}\right)$

$\operatorname{ASC}\left(m^{2}\right)$

PAS (mm Hg)

PAD $(\mathrm{mm} \mathrm{Hg})$

$70,0 *$

$(60,0-73,7)^{+}$

\section{Características Ecocardiográficas}

Estructura y Función Sistólica del Ventrículo Izquierdo

Septum interventricular $(\mathrm{mm})$

Pared posterior $(\mathrm{mm})$

VID diámetro $(\mathrm{mm})$

VIS diámetro (mm)

Masa (g)

Masa índex $\left(\mathrm{g} / \mathrm{m}^{2}\right)$

62,7

VID vol. (cc)

VID índex $\left(\mathrm{cc} / \mathrm{m}^{2}\right)$

42,7

VIS (cc)

FEVI (\%)

60,8

Función Diastólica del Ventrículo Izquierdo

$\mathrm{E}(\mathrm{cm} / \mathrm{s})$

$A(\mathrm{~cm} / \mathrm{s})$

$E / A$

TD (ms)

$\mathrm{e}^{\prime}$ septal $(\mathrm{cm} / \mathrm{s})$

$\mathrm{e}^{\prime}$ lateral $(\mathrm{cm} / \mathrm{s})$

Ventrículo Derecho y Presión Sistólica de Arteria Pulmonar

VD basal $(\mathrm{mm})$

TAPSE $(\mathrm{mm})$

FAC VD (\%)

TAP (ms)

PSAP (mmHg)

Volumen de la Aurícula Izquierda

Vol Al index $\left(\mathrm{cc} / \mathrm{m}^{2}\right)$
64

32,0

3,8

56

23,9

2,6

44

50,4

8,5

63

145,3

19,2

24,1

9,2

56

\section{6,8}

* Mediana +Intervalo intercuartil IMC: Índice de masa corporal, ASC: Área de superficie corporal, PAS: Presión arterial sistólica, PAD: Presión arterial diastólica VID: ventrículo izquierdo en fin de diástole, VIS: ventrículo izquierdo en fin de sístole, FEVI: fracción de eyección del ventrículo izquierdo, TD: tiempo de desaceleración de la onda E, VD: ventrículo derecho, TAPSE: excursión sistólica del anillo tricúspideo, FAC: fracción de área de acortamiento, TAP: tiempo de aceleración pulmonar, PSAP: presión sistólica de arteria pulmonar, Al; aurícula izquierda 
El Comité de Ética Institucional (IETSI) aprobó el presente estudio. Todos los participantes firmaron el consentimiento informado por escrito antes de ser reclutados en el estudio y se mantuvo la confidencialidad de sus datos.

Se estudiaron un total de 64 sujetos recuperados de infección por SARS-CoV-2, en los 3 a 6 meses luego de la enfermedad activa. La edad promedio fue 37,7 años $\pm 9,9$ años, y el $70 \%$ fueron del sexo femenino (Tabla 1). El personal de tipo asistencial fue el grupo predominante $(80 \%)$.

Dentro de las principales comorbilidades, la obesidad fue la más prevalente $(13,8 \%)$, seguida por hipertensión arterial $(4,6 \%)$ y diabetes mellitus tipo 2 (3,1\%). Ello muestra una población mayoritariamente libre de condiciones que puedan impactar sobre la estructura o función cardíaca.

El diagnóstico de infección por SARS-COV-2 fue realizado en el $60 \%$ de los casos con prueba serológica, en más del $50 \%$ por prueba molecular y solo 1,5\% tuvo diagnóstico tomográfico. La población de estudio principalmente presentó enfermedad leve (85\%) y menos de $5 \%$ cursó con enfermedad severa.

En relación a los hallazgos ecocardiográficos del ventrículo izquierdo, los promedios del grosor de pared, diámetros diastólico y sistólico, volumen diastólico y masa indexada del ventrículo izquierdo se encontraron dentro de rangos conservados. Asimismo, la media de la fracción de eyección de esta cavidad fue normal $(60 \% \pm 4,48)$. En la valoración de la función diastólica del ventrículo izquierdo, se evidenció que la relación de las ondas diastólicas con las presiones de llenado ventricular, se mantenían en rangos adecuados. Estos resultados concuerdan con los hallazgos de Sechi et al. ${ }^{[5]}$ quienes al seguimiento no hallaron alteraciones estructurales ni funcionales del ventrículo izquierdo, aun en casos severos. Es importante mencionar que Churchill et al., encontraron que el $26 \%$ de pacientes críticos presentaron disfunción sistólica del ventrículo izquierdo, lo cual revirtió en más del $80 \%$ a los 15 días. Los hallazgos en fase aguda se explican por la miocarditis potencial originada en la respuesta inflamatoria sistémica al SARS-COV-2, y su mayoritaria reversión en el seguimiento es afín a los datos obtenidos en nuestro estudio. ${ }^{[6]}$

Los mecanismos patológicos trombóticos descritos sobre la circulación pulmonar hacen la exploración del ventrículo derecho de gran interés. Al respecto, nuestros resultados demostraron que el diámetro basal $(32 \mathrm{~mm})$ y las variables subrogadas de la función sistólica como TAPSE $(23.6 \mathrm{~mm})$ y el cambio de área fraccional (FAC) (50\%) resultaron dentro de límites normales. En relación con la hipertensión pulmonar, el $100 \%$ de sujetos se categorizó como Baja Probabilidad de Hipertensión pulmonar. Asimismo, la variable Tiempo de aceleración pulmonar (TAP), de demostrado valor específico para evaluar resistencias vasculares pulmonares, se encontró en rango normal (145.3ms). Estos datos se relacionan con los hallazgos de Sechi et al., y Catena et al. ${ }^{[5,7]}$, quienes en el seguimiento de pacientes que tuvieron enfermedad severa o elevación de troponinas, no evidenciaron hallazgos patológicos del ventrículo derecho. La resolución de los factores patogénicos inflamatorios y protrombóticos favorecerían en mantener el ventrículo derecho sin alteraciones estructurales o funcionales en los controles.

En cuanto a la presencia de valvulopatías, ninguno de los sujetos presentó alteraciones valvulares mayores a regurgitación tricúspidea leve. Asimismo, en el $100 \%$ de los pacientes se descartó la presencia de derrame pericárdico.

Nuestros resultados muestran que los pacientes recuperados de infección leve por SARS-CoV-2, no presentan alteraciones cardíacas estructurales o funcionales en la exploración ecocardiográfica convencional. Aún así, consideramos importante determinar hallazgos estructurales más sutiles por medio de otros métodos, como la Deformación Miocárdica o la Resonancia Magnética, y realizar estudios de seguimiento a largo plazo.

Fuente de financiamiento: Autofinanciado

Conflicto de intereses: Los autores declaran no tener ningún conflicto de interés relevante

\section{ORCID}

Baltodano Arellano Roberto, https://orcid.org/0000-0002-75382125

Kelly Cupe Chacalcaje, https://orcid.org/0000-0002-7497-3898

Paol Rojas, https://orcid.org/0000-0002-2652-211X

Giovanni Meneses, https://orcid.org/0000-0003-1677-3457

Dante Urdanivia-Ruiz, https://orcid.org/0000-0002-1061-3893

Eliana Rafael-Horna, https://orcid.org/0000-0002-3670-0776

Luis Falcón-Quispe, https://orcid.org/0000-0002-7055-2308

Angela Cachicatari-Beltrán, https://orcid.org/0000-0001-7908 5200

Karla Sue América Hurtado-Belizario, https://orcid.org/00000001-6981-6540

Gerald Levano-Pachas, https://orcid.org/0000-0002-4136-582

\section{REFERENCIAS BIBLIOGRÁFICAS}

1. Zhu N, Zhang D, Wang W, Li X, Yang B, Song J et al. A novel coronavirus from patients with pneumoniain China, 2019. N Engl J Med 382:727-733. Disponible en: https ://doi.org/10.1056/NEJMo a2001017

2. Guo T, Fan Y, Chen M, Wu X, Zhang L, He T et al. Cardiovascular Implications of Fatal Outcomes of Patients with Coronavirus Disease 2019 (COVID-19). JAMA Cardiol. 2020 Jul 1;5(7):811-818. doi: 10.1001/jamacardio.2020.1017. Erratum in: JAMA Cardiol. 2020 Jul 1;5(7):848. PMID: 32219356; PMCID: PMC7101506.

3. Puntmann VO, Carerj ML, Wieters I, Fahim M, Arendt C, Hoffmann $J$ et al. Outcomes of Cardiovascular Magnetic Resonance Imaging in Patients Recently Recovered from Coronavirus Disease 2019 (COVID-19). JAMA Cardiol. 2020 Nov 1;5(11):1265-1273. doi: 10.1001/jamacardio.2020.3557. Erratum in: JAMA Cardiol. 2020 Nov 1;5(11):1308. PMID: 32730619; PMCID: PMC7385689.

4. Szekely Y, Lichter Y, Taieb P, Banai A, Hochstadt A, Merdler I et al. Spectrum of Cardiac Manifestations in COVID-19: A Systematic 
Echocardiographic Study. Circulation. 2020 Jul 28;142(4):342-353. doi: 10.1161/CIRCULATIONAHA.120.047971. Epub 2020 May 29. PMID: 32469253; PMCID: PMC7382541.

5. Sechi LA, Colussi G, Bulfone L, Brosolo G, Da Porto A, Peghin M et al. Short-term cardiac outcome in survivors of COVID-19: a systematic study after hospital discharge. Clin Res Cardiol. 2021 Jul;110(7):1063-1072. doi: 10.1007/s00392-020-01800-z. Epub 2021 Jan 22. PMID: 33481096; PMCID: PMC7820534.

6. Churchill T, Bertrand P, Bernard S, Namasivayam M, Churchill J, Crousillat D et al. Echocardiographic Features of COVID-19 Illness and Association with Cardiac Biomarkers. Journal of the American Society of Echocardiography. Disponible en doi: 10.1016/j. echo.2020.05.028

7. Catena C, Colussi G, Bulfone L, Da Porto A, Tascini C, Sechi LA. Echocardiographic Comparison of COVID-19 Patients with or without Prior Biochemical Evidence of Cardiac Injury after Recovery. J Am Soc Echocardiogr. 2021 Feb;34(2):193-195. doi: 10.1016/j. echo.2020.10.009. Epub 2020 Oct 22. PMID: 33243582; PMCID: PMC7580530 